\title{
Nutritional influences on the outcome of selection
}

\section{By D. S. Falconer, A.R.C. Unit of Animal Genetics, Department of Genetics, University of Edinburgh, Edinburgh $E H_{9} 3 \mathfrak{F} \mathrm{N}$}

My object in this paper is to revive an old problem in the genetics of selective breeding and to show where nutritional physiology may perhaps be able to help towards a solution. The general problem is this. If you measure some character, e.g. growth rate, in two different environments, e.g., dietary regimen, is the genetic variation in the two environments caused by the same or by different genes? Do the genes that cause good growth in a good environment also cause good growth in a bad environment, or are different genes required? The answer is now clear: some, but not all, of the desirable genes are different. Good growth on different levels of nutrition would be expected to require somewhat different physiological properties, and to be to that extent genetically distinct. The problem then is what are the different sorts of gene doing; what are the physiological differences involved? To understand these differences is important practically because we may be able to influence the outcome of selective breeding by choosing the right environment, for example to produce animals that grow well without becoming fat. The experiments that we did with mice are, in outline, as follows.

We measured growth from weaning ( 3 weeks) to sexual maturity (6 weeks), and we fed the mice during this period on two different diets. The 'full' diet was the normal commercial pellets ad lib. and the 'restricted' diet was a reduced intake of the same food. In the first experiment (Falconer \& Latyszewski, 1952), the restriction was achieved by housing the mice singly and giving them a weighed quantity of the pellets daily, calculated to be about $75 \%$ of the normal, ad lib. intake. This was a laborious procedure and for the second experiment (Falconer, 1960) a special pellet was made of the normal ingredients diluted with $50 \%$ of indigestible fibre, and this was given ad lib. The mice did not eat the fibre; feeding required more effort on their part and the intake was reduced to about $80 \%$ of normal. The two methods of restriction were, of course, not equivalent. In particular the second method allowed expression of natural variation of 'appetite', i.e., the inclination of the mouse to work for its living, which the first did not.

We selected for increased growth rate in two separate lines, one kept on full diet and the other on restricted diet, from 3 to 6 weeks of age. In both we got an improvement of growth. At the end, or in the second experiment in every generation, we reared some mice of each line on the other diet, to see how much of the improvement would be carried over. This would represent 'common ground' between the two characters. In genetic terms the similarity can be expressed as the 
genetic correlation between growth on full and growth on restricted diets. Estimated from the first four generations of the second experiment, the genetic correlation was 0.66 , which means that $44 \%$ (i.e., $r^{2}$ ) of the genetic variance is common to the characters, with $56 \%$ being particular to the specific environment. There have been some subsequent experiments by others which agree in showing that growth rates on different diets are to some extent under separate control; for example Korkman (196I) with mice; Park, Hansen, Chung \& Chapman (1966) with rats; and Fowler \& Ensminger (1960) with pigs.

I shall return to the genetic correlation with its physiological implications. First I must describe a puzzling feature of the experiments, particularly the second one when we looked at what thirteen generations of selection had produced.

When the genetic correlation is less than unity but greater than zero, one would expect a partial carry-over of the improvement made by selection from one diet to the other. Mice selected for growth on full diet, for example, should show some improvement of growth on restricted diet, but not as much improvement as in the mice selected on restricted diet. To generalize, and I have no doubt that this is a valid generalization, if you want to improve animals for performance in a specific environment you should do the selection in that environment and not in some other. (Precise prediction of the amount of carry-over is rather technical, depending on the two heritabilities and intensities of selection as well as the genetic correlation.) What we found, however, did not agree with this general prediction. The line selected for growth on full diet was improved by about $40 \%$, growth on full diet having increased from about ${ }_{13}$ to about $18 \mathrm{~g}$. When contemporary mice of this line were reared on the restricted diet, however, their growth was not at all improved over that at the beginning, so there appeared to be no carry-over of the improvement from full to restricted diet. With the other line quite the reverse happended. Selection on restricted diet led to an improvement of about $30 \%$, growth on restricted diet having increased from about I I to about $\mathrm{x} 4 \mathrm{~g}$. When the mice of this line were reared on full diet their growth was fully as good as that of the line selected for growth on a full diet. There appeared to be complete carry-over of the improvement. These results do not make sense genetically. They are contradictory, since one line indicated a very low genetic correlation, and the other a very high one. (The value of 0.66 given earlier is based on the first four generations and is a sort of average taking account of the other factors involved, which need not be elaborated here.) Both of my experiments showed the same anomalous asymmetrical carry-over, but the other experiments cited earlier did not, or at least not nearly to the same degree. The genetical problem of my experiments is still unsolved. I do not think the asymmetrical carry-over can be accounted for in physiological terms because the inconsistency will not be removed by translation into another language. I now think that the most likely explanation is random genetic change due to the small number of parents used. This random genetic drift can cause quite large differences between different lines selected in exactly the same way (Falconer, 1973). If the asymmetrical carry-over were a general phenomenon, and not accidental, it would be important for breeding 
practice. It would indicate selection in the worst environment as the best way to improve animals for performance in a range of different environments.

The idea of genetic correlation implies a distinction between three classes of gene contributing to the variation of growth between individuals. Genes of Class A, say, affect growth on full diet, but do not affect growth on restricted diet; they contribute to variation in the former environment but not in the latter. Class B do the opposite; they affect growth on restricted diet but not on full diet. Class $\mathrm{C}$, the common component, affect growth on both diets. We would like to know what are the physiological attributes influenced by these three classes of gene. We have made a few simple comparisons between the two selected lines, which go a little way towards showing how the genes of Classes $A$ and $B$ differ in their effects.

Table I. Growth, food intake and efficiency of male mice from 3 to 6 weeks of age

No. of mice

Gain in weight (g)

Food consumed (g)

Efficiency (g)

Food wasted (g)

Food wasted (\%)

$$
\text { (Efficiency }=\text { gain in weight } \div \text { food consumed) }
$$

\begin{tabular}{|c|c|c|c|c|}
\hline & \multicolumn{2}{|c|}{ Grown on full diet } & \multicolumn{2}{|c|}{ Grown on restricted diet } \\
\hline & 'Full-line' & 'Restr.-line' & 'Full-line' & 'Restr.-line' \\
\hline $\begin{array}{l}\text { No. of mice } \\
\text { Gain in weight (g) } \\
\text { Food consumed (g) } \\
\text { Efficiency (g) } \\
\text { Food wasted (g) } \\
\text { Food wasted (\%) }\end{array}$ & $\begin{array}{r}5 \\
20 \cdot 1 \\
111 \cdot 0 \\
18 \cdot 7 \\
16 \cdot 1 \\
12 \cdot 7^{\circ 0}\end{array}$ & $\begin{array}{r}5 \\
19 \cdot 0 \\
116 \cdot 1 \\
16 \cdot 4 \\
9 \cdot 7 \\
7 \cdot 7\end{array}$ & $\begin{array}{r}4 \\
1 \cdot 7^{\circ} \\
67 \cdot 3^{\circ} \\
2 \cdot 6^{\circ} \\
124 \cdot 3 \\
64 \cdot 9^{\circ}\end{array}$ & $\begin{array}{r}4 \\
11 \cdot 1 \\
109 \cdot 7 \\
9 \cdot 3 \\
92 \cdot 8 \\
45 \cdot 8\end{array}$ \\
\hline
\end{tabular}

Significance levels of differences between lines: $\bullet \bullet \bullet<0.01 ; \bullet P<0.02 ; \bullet P<0.05$.

After the selection in the second experiment was stopped at generation 13 we measured individual food intake and growth of both lines on both diets, and we measured the carcass composition of both lines when grown on the full diet. For the measurement of food intake the mice, all males, were caged separately from 3 to 6 weeks of age. At intervals of 2 or $3 \mathrm{~d}$ the amount of food consumed was measured and also the amount wasted by scattering on the floor of the cage. The totals over the 3-week period are given in Table 1 . When grown on the full diet the two lines did not differ in weight gain, in food intake or in efficiency. They did, however, differ in the amount and proportion of food wasted. The line selected on full diet scattered nearly twice as much food on the floor of the cage as did the line selected on restricted diet. From other evidence it is probable that the major change in feeding habits was in the restricted-diet line, which the selection had made less prone to drop crumbs on the floor. When grown on restricted diet the two lines differed in gain, food intake and efficiency, and also in their wastage of food. To take the food wastage first: the diet contained $50 \%$ of fibre in the form of ground oat husks. The restricted-diet line wasted $46 \%$ of the food removed from the container. Most of the waste could be seen to be fibre which was presumably rejected deliberately. The full-diet line wasted $65 \%$, so the mice must have rejected a good deal of the edible with the inedible. The difference between the two lines $36(1)$ 
suggests strongly that the change in feeding habits in the restricted-diet line must have been a contributory cause of the increased growth on restricted diet. Perhaps their greater skill in feeding selectively reduced the time and effort needed to ingest a given amount of food and the reduced effort led to an increased intake and faster growth.

It is tempting to conclude that the higher efficiency of the restricted-diet line was a cause of its better growth on restricted diet, but I do not think this conclusion is justified, for two reasons. First, the efficiency was not higher on full diet. Second, and more important, efficiency was correlated with gain and all lines (including others not mentioned here) on both diets fitted more or less on the same regression line; when the two lines on restricted diet were adjusted by regression to an equal gain, they did not differ in efficiency.

The fatness of the two lines when reared on full diet was compared. First the abdominal fat was measured on several samples of mice from both experiments. All showed the restricted-diet line to have less fat than the full-diet line. The abdominal fat as a percentage of body-weight was as follows. In the first experiment, males aged 6 weeks, full-diet line $2.0 \%$, restricted diet line $1 \cdot 4 \%$; in the second experiment, males aged 12 weeks, full-diet line $2.0 \%$, restricted diet line $1.5 \%$. Second, total fat was estimated by carcass analysis in some of the mice aged 12 weeks. The results are given in Table 2 . The restricted-diet line had less fat and more protein as indicated by water. The difference in percentage of fat was not significant but the difference in percentage water was $(P<0.05)$. Thus a clear difference was that selection on the restricted diet produced mice that diverted less of their intake to fat. This allowed better growth because, presumably, of the higher energy content of fat than of protein. The reduced diversion to fat was not just a response to the restricted food intake because it remained when intake was not restricted.

Table 2. Carcass composition of male mice aged 12 weeks grown on full diet

No. of mice
Body-weight $(\mathrm{g})$
Carcass weight $(\mathrm{g})$
Total fat $(\mathrm{g})$
Water $(\mathrm{g})$
Fat $(\%$ of carcass weight)
Water (\% of carcass weight)

\begin{tabular}{|c|c|}
\hline \multicolumn{2}{|c|}{ Line selected on } \\
\hline Full diet & Restricted diet \\
\hline 8 & 7 \\
\hline $\begin{array}{l}43 \cdot 1 \\
34 \cdot 2\end{array}$ & $\begin{array}{l}47 \cdot 8 \\
38 \cdot 5\end{array}$ \\
\hline $\begin{array}{r}5 \cdot 5 \\
20 \cdot 2\end{array}$ & $\begin{array}{r}4 \cdot 5 \\
24.4\end{array}$ \\
\hline $\begin{array}{l}15.4 \\
59.6\end{array}$ & $\begin{array}{l}11 \cdot 8 \\
63.4\end{array}$ \\
\hline
\end{tabular}

These comparisons of the lines unfortunately do not go far towards identifying the effects of the genes in the three classes. The asymmetrical carry-over only allows us to distinguish Class $B$ from the others. The comparison of the two lines has identified two physiological effects of genes in Class B, i.e., of genes affecting growth on restricted diet but not on full diet. These are the more economical feeding habits and the reduced proportion of fat in the carcass. 
REFERENCES

Falconer, D. S. (1960). Genet. Res. 1, 91

Falconer, D. S. (1973). Genet. Res. 22, 291.

Falconer, D. S. \& Latyszewski, M. (1952). J. Genet. 51, 67.

Fowler, S. H. \& Ensminger, M. E. (1960). J. Anim. Sci. 19, 434.

Korkman, N. (1961). Hereditas 47, 342.

Park, Y. I., Hansen, C. T., Chung, C. S. \& Chapman, A. B. (1966). Genetics 54, I315. 